\title{
Using aquablation to treat lower urinary tract symptoms in benign prostatic hyperplasia.
}

\author{
KENNEDY, C., PATERSON, C.
}

2020 


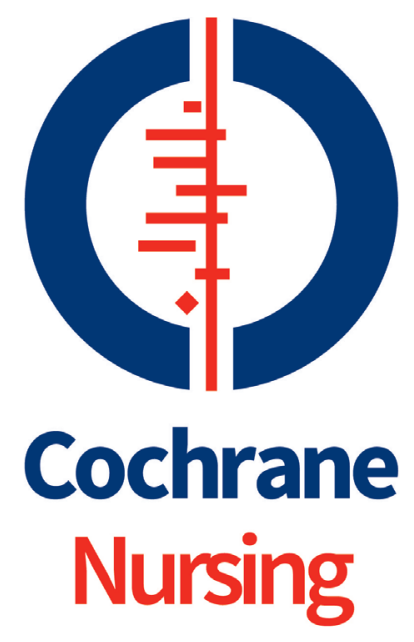

Cochrane Nursing - Cochrane Review Summary

Prepared for the

\section{American Journal of Nursing}

\section{TITLE: Aquablation of the prostate for the treatment of lower urinary tract symptoms in men with benign prostatic hyperplasia}

Cochrane Corner Writer:

Catriona Kennedy* PhD, BA (Hons) Dip Nurs. RN, DN PG Cert RNT

QNIS Professor of Community Nursing

School of Nursing and Midwifery, Robert Gordon University Aberdeen Scotland.

c.m.kennedy1@rgu.ac.uk

Catherine Paterson* PhD, BA, MSc, RAN, PG Cert LTA, FHEA

Associate Professor in Nursing

School of Nursing, Midwifery and Public Health, University of Canberra, ACT, Australia Catherine.paterson@canberra.edu.au

${ }^{*} \mathrm{~A}$ member of the Cochrane Nursing $(\mathrm{CN})$ 


\section{- Background:}

The prostate is normally a walnut-sized gland located just below the urinary bladder. The prostate encircles the neck of the bladder, thus it can affect urine flow. Benign prostatic hyperplasia (BPH) may cause prostatic enlargement and subsequently compression of the urethra and can cause urinary obstruction. BPH is of clinical significance when men experience bothersome lower urinary tract symptoms (LUTS). LUTS can cause distress and negatively impact on quality of life (QOL) thus requiring treatment and intervention.

Shared treatment decisions are based upon the level of distress, bother, severity and burden of LUTS on the patients QOL. Initial treatment options for BPH include conservative management (watchful waiting and lifestyle modification) and medications (alpha-blockers and 5-alpha reductase inhibitors). However, if patients experience complications such as acute urinary retention, recurrent urinary tract infection, bladder stones or diverticula, haematuria, or renal insufficiency or don't respond to medical treatment then surgical options are considered. Clinical guidelines recommend monopolar or bipolar transurethral resection of the prostate (TURP) as the first line surgical approach. It is regarded as an effective form of treatment that all urologists are trained to perform. However, TURP can cause complications, such as bleeding, urinary tract infections, bladder neck contracture, erectile dysfunction and retrograde ejaculation (more than $50 \%$ of patients). It can also in rare instances cause dilutional hyponatraemia (known as TUR syndrome). To avoid these issues, newer minimally invasive surgical alternatives are being developed and starting to be used in practice, such as Aquablation. Aquablation is a minimally invasive water ablation therapy which combines image guidance and robotics for the removal of benign prostatic tissue. However, it is unclear if Aquablation translates to similar or improved clinical outcomes with fewer complications compared to traditional surgical approaches.

\section{- Objective/s:}

The overall aim was to assess the effectiveness of Aquablation for the treatment of lower urinary tract symptoms in men with $\mathrm{BPH}$. The primary outcomes were urologic scores, QoL and major adverse events (Hwang et al 2019). Other outcomes of interest were retreatments, erectile function and ejaculatory dysfunction. 
Participants in parallel group randomised controlled trials, cluster-randomised trials and prospective cohort studies with concurrent comparison groups were men (age over 40) diagnosed with BPH (prostate volume of $20 \mathrm{~mL}$ or greater, International Prostate Symptom Scores (IPSS) of eight or over, maximum flow rate of less than $15 \mathrm{~mL} /$ second). Studies eligible for inclusion used either chance to decide how men were treated or studies where the urologist determined the treatment. Studies compared Aquablation (experimental intervention) with other procedures to manage urinary symptoms caused by an enlarged prostate up to 12 months of follow up or less.

\section{- Intervention/Methods:}

Aquablation is a minimally invasive therapy using water ablation in combination with image guidance and robotics for the removal of prostatic tissue. This therapy delivers a high-pressure water jet, which can ablate the adenomatous tissue whilst preserving collagenous structures such as the surgical capsule, bladder neck and blood vessels.

Comparisons of Aquablation against sham control or no intervention, transurethral resection of the prostate (TURP), laser ablations or enucleations of the prostate, prostatic urethral lift, convective radiofrequency, other minimally invasive therapies or simple prostatectomy were the focus. The researchers sought to determine whether Aquablation was more effective up to 12 months follow up than other interventions through mean changes from baseline or final mean value in urologic symptom scores using a validated scale such as the IPSS.

\section{- Results:}

One single multicentre (17 centers) and multicountry (Australia 1, New Zealand 1,UK 3 and USA 12 all classed as high income countries) RCT was included. A total of 184 participants (of which 168 were white) were involved in this RCT which was sponsored by the device manufacturer. 
This study compared Aquablation to TURP to 12 months follow up. Change in IPSS were measured at baseline, I month, 3 months, 6 months and 12 months. Overall the reviewers found that Aquablation probably has similar results to TURP for urological scores and may improve QoL. The evidence is uncertain about impact on the risk of adverse events. Aquablation (up to 12 months) may offer similar rates of retreatment, erectile and ejaculatory dysfunction to TURP but the evidence is very uncertain. The authors state there may be a small benefit to sexually active men in ejaculatory function but this is uncertain. The study was not designed to assess subgroup effects so the limited evidence that Aquablation may improve urologic symptom scores in men with a prostate volume of $50 \mathrm{~mL}$ or above should be treated with caution.

\section{- Conclusions:}

The authors of this review conclude that the effects of Aquablation over TURP on urologic symptom scores are probably similar, and QoL may be similar at 12 months. The reviewers were unable to measure adverse events with concerns over imprecision and selective reporting bias and therefore it is very uncertain whether these are similar. The one included RCT was judged to be moderate to very low certainty evidence depending on the outcome although 12 months is quite a short follow up period. The reviewers identified that patients and clinicians making decisions about treatment options needs to be aware that the evidence for Aquablation is drawn from a single, relatively small RCT supported by the device maker. The present evidence does not support the use of Aquablation to other treatment forms in men with lower urinary tract symptoms due to $\mathrm{BPH}$. More rigorous and transparently reported research is required as the current evidence base offers little guidance for nurses as to alternative effective interventions for $\mathrm{BPH}$. Future research should compare Aquablation to a range of treatment forms alongside the collection of longer-term data. Overall certainty about the evidence in this review ranged from moderate to very low however, the high degree of uncertainty with regard to harm means we can have little confidence in these results for clinical decision-making.

\section{- Implications for Practice:}

$\mathrm{BPH}$ is a common condition in men over the age of 50 years old. BPH can cause debilitating and distressing LUTS which can profoundly affect QOL. Nurses can 
support patients by developing shared self-management care plans, particularly around the conservative management options, including lifestyle modifications (i.e caffeine reduction, bladder retraining, fluid intake, bowel habits, pelvic floor exercises, and smoking cessation) and patient education and counselling for medications (alpha-blockers and 5-alpha reductase inhibitors). However, for patients who require surgical intervention for the treatment of $\mathrm{BPH}$ this review has several important considerations for nursing practice.

Given the dearth of research which compared Aquablation to traditional surgical approaches for $\mathrm{BPH}$ we are not clear about the benefits or indeed the potential harms of this new minimally invasive surgical approach. Nurses are fully embedded in the multidisciplinary team and can be involved in supporting treatment decisions for the clinical management of $\mathrm{BPH}$, and co-ordinate referrals to their urologists for consideration of surgical intervention for patients. Nurses should clearly explain that there is a lack of robust empirical evidence to recommend Aquablation at this time compared to traditional surgical techniques.

Internationally, given the different funding models within healthcare systems patients may also be at risk of financial toxicity in relation to Aquablation. Nurses should be aware of this significant issue that may impact upon patients. Based upon the results of one study, at this time nurses are unable to recommend this treatment as evidence-based practice for patients affected by $\mathrm{BPH}$ in our care.

\section{- References:}

Hwang EC, Jung JH, Borofsky M, Kim MH, Dahm P. Aquablation of the prostate for the treatment of lower urinary tract symptoms in men with benign prostatic hyperplasia.

Cochrane Database of Systematic Reviews 2019, Issue 2. Art. No.: CD013143.

DOI: 10.1002/14651858.CD013143.pub2. 